\title{
FATORES RELACIONADOS À OCORRÊNCIA DE QUEDA DE IDOSOS
}

Luiz Alberto BEIJO ${ }^{1}$

Fabrício Goecking AVELAR ${ }^{2}$

Carlos José dos REIS ${ }^{3}$

Larissa da Costa TEODORO ${ }^{4}$

Sueli Leiko Takamatsu GOYATÁ ${ }^{5}$

Matheus Pereira ARAÚJO ${ }^{6}$

\footnotetext{
${ }^{1}$ Doutor, Docente do instituto de Ciências Exatas. Universidade Federal de Alfenas. luiz.beijo@unifal-mg.edu.br

${ }^{2}$ Doutor, Docente do instituto de Ciências Exatas. Universidade Federal de Alfenas. fabricioavelar@yahoo.com.br

${ }^{3}$ Acadêmico do curso de Matemática. Universidade Federal de Alfenas. carlosjreis17@yahoo.com.br

${ }^{4}$ Acadêmica do curso de Biotecnologia. Universidade Federal de Alfenas. larissa_teodoro_ifsemg@hotmail.com

${ }^{5}$ Doutora, Docente da Escola de Enfermagem. Universidade Federal de Alfenas. sueligoyata@yahoo.com.br

${ }^{6}$ Acadêmico do curso de Farmácia. Universidade Federal de Alfenas. araujo88@ hotmail.com
}

Recebido em: 04/04/2016 - Aprovado em: 09/01/2017 - Disponibilizado em: 01/07/2017

\begin{abstract}
RESUMO:
A população idosa brasileira está crescendo acarretando o aumento da preocupação com este contingente populacional. Por essa razão é necessário identificar os principais fatores de risco da população idosa a fim de subsidiar o planejamento das ações desenvolvidas pelos órgãos de saúde. O estudo teve como objetivo verificar se fatores fisiológicos, ambientais e cognitivos influenciam na ocorrência de quedas de idosos. Para a coleta de dados foram aplicados questionários estruturados em visitas domiciliar realizadas no período de abril de 2011 a setembro de 2012 com 78 idosos da cidade de Alfenas- MG. Verificou-se que variável sexo não interfere significativamente no risco de quedas em idosos. Nenhum dos fatores fisiológicos houve correlação significativa com as quedas. Sofreram queda no período $32,1 \%$ dos entrevistados. O modelo de regressão logística foi ajustado aos dados. Foram significativos o histórico de quedas anteriores, com razão de chance de 6,4; a idade, com razão de chance de 8,2; e a presença de tapetes espalhados pelo chão com razão de chance de 4,4. Logo, existe a necessidade de medidas de atenção básica a esses fatores por parte de programas assistenciais de saúde para reduzir a ocorrência de quedas de idosos.

Palavras-chave: Medicamentos de controle especial. Acidentes por quedas. Saúde do idoso. Serviços de saúde para idosos. Histórico de quedas.
\end{abstract}

\section{FACTORS RELATED TO THE OCCURRENCE OF FALLS IN ELDERLY}

\begin{abstract}
:
The elderly population is growing and will be more than 30 million people in 20 years, causing increasing the concern for this population. For this reason, it is necessary to identify the main risk factors to elderly population in order to support the planning of actions undertaken by health agencies. The study aimed to verify if the variables, sex, use of prescription drugs, history of falls and physiological factors influence the risk of falling elderly. For data collection it was administered structured questionnaires during home visits conducted from April 2011 to September 2012 with 78 elderly from the city of Alfenas at Minas Gerais State. It was found that the gender variable does not influence the risk of falls in the elderly. None of the physiological factors correlate significantly with the falls presented by the interviewees. Suffered falls in the period $32.1 \%$ of respondents. The regression model Logistic was fitted to the data. Were signicant the history of previous falls, with an odds ratio of 6.4; age, with an odds ratio of 8.2 ; and the presence of rugs scattered on the floor with an odds ratio of 4.4. Therefore, there is need for measures of primary care to these factors by health care programs to reduce the occurrence of falls in the elderly.
\end{abstract}

Keyword: Controlled drugs. Accidental falls. Elderly health. Health services for the elderly. History of falls 


\section{INTRODUÇÃO}

O envelhecimento populacional tem ganhado reconhecimento universal e está ocorrendo tanto em países em desenvolvimento quanto em desenvolvidos. $\mathrm{O}$ aumento da expectativa de vida, no Brasil, tem feito com que população idosa brasileira cresça em ritmo acelerado. Nesse contexto, é necessário preocupar-se com esse contingente populacional, pois os idosos apresentam condições específicas que promovem o aumento da vulnerabilidade quanto às perdas, tanto no ponto de vista funcional como emocional, bem como social e econômico. Essas condições os predispõem, principalmente, à presença de múltiplas doenças, baixa auto-estima, depressão, incapacidade para realizações das atividades cotidianas e pobreza, promovendo grandes interferências na qualidade de vida dessa população. Novas políticas de saúde voltadas à população idosa, referentes às ações estratégias de prevenção e atenção integral no cuidado à saúde do idoso fazem-se necessárias (CARVALHO; LUCKOW; SIQUEIRA, 2011; VERAS, 2009).

O Brasil ocupa hoje a sétima colocação mundial em número de idosos, e segundo a Organização Mundial de Saúde (OMS), até 2025, o Brasil ocupará a sexta posição (INSTITUTO BRASILEIRO DE GEOGRAFIA E ESTATÍSTICA, 2010). Só em 2010, no Brasil, o número de pessoas com mais de 60 anos já ultrapassava 20 milhões, se aproximando do número de crianças com até nove anos de idade (BRASIL, 2012). Diante desse cenário, faz-se necessário identificar os principais fatores de risco da população idosa a fim de subsidiar o planejamento das ações desenvolvidas pelos órgãos de saúde, com o intuito de proporcionar melhores condições para uma boa qualidade de vida e evitando, consequente, a diminuição da capacidade funcional e cognitiva dessas pessoas (CARVALHO; LUCKOW; SIQUEIRA, 2011; INSTITUTO BRASILEIRO DE GEOGRAFIA E ESTATÍSTICA， 2010; COUTINHO; SILVA, 2002).

O envelhecimento é um processo dinâmico, progressivo e fisiológico, acompanhado por modificações morfológicas e funcionais, assim como modificações bioquímicas e psicológicas, resultando na diminuição da reserva funcional dos órgãos e aparelhos (HORAK, 2006). A diminuição da velocidade de contração muscular, da amplitude de movimento, alterações visuais e auditivas, da força muscular e postural influenciam a mobilidade funcional e o déficit de equilíbrio em idosos (PINHO et al., 2012). Isso faz com que as quedas possam ocorrer com maior frequência nessa faixa etária.

No caso específico dos idosos as quedas são fatores de forte impacto, sendo as contusões e fraturas as lesões mais comuns (FREITAS et al., 2015). 
O evento queda pode ser definido como ocorrências de desequilíbrio que levam o idoso a cair, o que acomete cerca de $30 \%$ dos idosos brasileiros (PERRACINI; RAMOS, 2002; SIQUEIRA et al., 2011). Esse evento é reconhecido como um importante problema de saúde pública entre os idosos em decorrência da frequência, da morbidade e do elevado custo social e econômico decorrente das lesões provocadas por elas (COUTINHO; SILVA, 2002; FREITAS; $\quad$ SCHEICHER, 2008; NICOLUSSI et al., 2012).

São vários os fatores de risco de quedas para idosos. A etiologia da queda é normalmente multifatorial, e as causas podem ser intrínsecas e extrínsecas. Os fatores intrínsecos são relacionados a própria pessoa, a qual pode apresentar redução da função dos sistemas que compõem o controle postural, doenças crônicas, tais como hipertensão, diabetes e dislipidemias, transtornos cognitivos e comportamentais, apresentando incapacidade em manter ou para recuperar o equilíbrio. Como fatores extrínsecos podem citar-se o local em que vive, tais como iluminação, tapetes soltos, móveis em excesso (PINHO et al., 2012).

Devido às dificuldades de recuperação próprias dos idosos que sofrem quedas, o que provoca a redução da qualidade de vida do idoso e consequente aumento dos gastos públicos com a saúde, objetivou-se com esse trabalho identificar quais fatores podem influenciar no risco e queda dos idosos cadastrados na unidade de Saúde da Família (PSF) Vila Formosa, na cidade de Alfenas MG. Foi avaliada a associação de características gerais dos idosos, fatores cognitivos, fisiológicos e ambientais com a ocorrência de quedas.

\section{METODOLOGIA}

Para a coleta de dados foram aplicados questionários estruturados, em visita domiciliar realizadas no período de julho de 2011 a julho de 2012, com 78 idosos (com idade de 60 anos ou mais e que assinaram previamente o Termo de Consentimento Livre e Esclarecido) escolhidos aleatoriamente nos cadastrados da unidade de Saúde da Família (PSF) Vila Formosa, da cidade de Alfenas MG, e que residem na área de abrangência da cidade de Alfenas.

Foi elaborado um instrumento contendo duas partes: a primeira sobre as características gerais dos idosos em relação à idade, sexo, se tomava ou não remédios controlados e outra contendo os fatores de risco do diagnóstico de enfermagem de Risco de Quedas:

a) Fatores Ambientais: móveis e objetos em excesso; ausência de material anti-derrapante no banheiro; Ausência de material antiderrapante no box do banheiro; ausência de material antiderrapante nos calçados; 
condições climáticas; mobilização; pouca iluminação no quarto de dormir; pouca iluminação no banheiro; quarto não familiar; tapetes espalhados pelo chão.

b) Fatores Cognitivos: estado mental rebaixado; história de quedas; idade igual ou acima de 65 anos; morar sozinho; demência; prótese de membro inferior; uso de cadeiras de roda; uso de dispositivos auxiliares

c) Fatores Fisiológicos: acidente vascular cerebral; doença neurológica; anemias; equilíbrio prejudicado; artrite; falta de sono; ansiedade; hipotensão ortostática; asma/bronquite; hipertensão arterial; artrose; incontinência urinária; condições pósoperatórias; incontinência fecal; constipação; insônia; déficits proprioceptivos; labirintite; força diminuída nas extremidades inferiores; mobilidade física prejudicada; depressão; diabetes mellitus; obesidade; dificuldade na marcha; osteoporose; dificuldades auditivas; obstrução pulmonar crônica; dificuldades visuais; problema de coluna; doença vascular; tontura; doença gastrointestinal; mudanças na taxa de açúcar após as refeições.

\section{Análise Estatística}

Pelo fato da variável resposta ser dicotômica (sim e não), os dados foram submetidos à análise pelo modelo de regressão logística e o ajuste foi realizado utilizando-se o software R. Para verificar a significância do modelo foi utilizado o teste de Qui-Quadrado (COLLET, 1991), este teste é comumente utilizado para verificar se o modelo é significativo ao nível $5 \%$ de probabilidade.

\section{RESULTADOS}

Este estudo envolveu 78 idosos acima de 60 anos, no qual, 41,00\% pertenciam ao sexo masculino e $59,00 \%$ ao sexo feminino. A idade do grupo variou entre 60 a 88 anos com média de 69,7 anos e desvio padrão de 6,9 anos. Foi verificado que $25(32,1 \%)$ do total de idosos apresentaram quedas durante o período de estudo. O uso de medicamentos controlados para a análise da influência no risco de queda em idosos. Do total de idosos entrevistados, $61,5 \%$ faziam uso de medicamentos controlados e destes, 15 $(31,2 \%)$ caíram. Dos que não faziam uso, nove $(32,1 \%)$ sofreram quedas (Tabela 1$)$. 
Tabela 1- Resultados entre a associação entre fatores de risco fisiológicos e a ocorrência de quedas de idosos

\begin{tabular}{|c|c|c|c|c|c|c|c|}
\hline \multirow{2}{*}{$\begin{array}{c}\text { Fator } \\
\text { Fisiológico }\end{array}$} & \multicolumn{2}{|c|}{ Queda } & \multirow[b]{2}{*}{ Total } & \multirow{2}{*}{$\begin{array}{c}\text { Fator } \\
\text { Fisiológico }\end{array}$} & \multicolumn{2}{|c|}{ Queda } & \multirow[t]{2}{*}{ Total } \\
\hline & $\begin{array}{l}\text { Não } \\
(\mathrm{n}=53)\end{array}$ & $\begin{array}{l}\text { Sim } \\
(n=25)\end{array}$ & & & $\begin{array}{l}\text { Não } \\
(n=53)\end{array}$ & $\begin{array}{l}\text { Sim } \\
(n=25)\end{array}$ & \\
\hline \multicolumn{4}{|l|}{ Idade (em anos) } & \multicolumn{4}{|c|}{ Doença neurológica } \\
\hline Média & 68,3 & 72,7 & 69,7 & Não & 53 & 23 & $76(97,4 \%)$ \\
\hline Desvio padrão & 6,9 & 6,2 & 6,9 & Sim & 0 & 2 & $2(2,6 \%)$ \\
\hline \multicolumn{4}{|l|}{ Sexo } & \multicolumn{4}{|c|}{ Mudanças na taxa de açúcar após as refeições } \\
\hline Masculino & 21 & 11 & $32(41,0 \%)$ & Não & 52 & 25 & $77(98,7 \%)$ \\
\hline Feminino & 32 & 14 & $46(59,0 \%)$ & Sim & 1 & 0 & $1(1,3 \%)$ \\
\hline \multicolumn{4}{|c|}{ Uso de remédios controlados } & \multicolumn{4}{|l|}{ Falta de sono } \\
\hline Não & 19 & 9 & $28(35,9 \%)$ & Não & 43 & 17 & $60(76,9 \%)$ \\
\hline Sim & 33 & 15 & $48(61,5 \%)$ & Sim & 10 & 8 & $18(23,1 \%)$ \\
\hline \multicolumn{4}{|c|}{ Acidente vascular cerebral } & \multicolumn{4}{|c|}{ Equilíbrio prejudicado } \\
\hline Não & 52 & 24 & $76(97,4 \%)$ & Não & 51 & 22 & $73(93,6 \%)$ \\
\hline Sim & 1 & 1 & $2(2,6 \%)$ & Sim & 2 & 3 & $5(6,4 \%)$ \\
\hline \multicolumn{4}{|l|}{ Anemias } & \multicolumn{4}{|c|}{ Hipertensão arterial } \\
\hline Não & 53 & 24 & $77(98,7 \%)$ & Não & 18 & 10 & $28(35,9 \%)$ \\
\hline Sim & 0 & 1 & $1(1,3 \%)$ & Sim & 35 & 15 & $50(64,1 \%)$ \\
\hline \multicolumn{4}{|l|}{ Artrite } & Hipotensão ortc & ática & & \\
\hline Não & 47 & 25 & $72(92,3 \%)$ & Não & 50 & 24 & $74(94,9 \%)$ \\
\hline Sim & 6 & 0 & $6(7,7 \%)$ & Sim & 3 & 1 & $4(5,1 \%)$ \\
\hline Ansiedade & & & & Incontinência u & ária & & \\
\hline Não & 40 & 14 & $54(69,2 \%)$ & Não & 51 & 21 & $72(92,3 \%)$ \\
\hline Sim & 13 & 11 & $24(30,8 \%)$ & Sim & 2 & 4 & $6(7,7 \%)$ \\
\hline Asma/Bronquite & & & & Incontinência f & & & \\
\hline Não & 46 & 19 & $65(83,3 \%)$ & Não & 52 & 23 & $75(96,2 \%)$ \\
\hline Sim & 7 & 6 & $13(16,7 \%)$ & Sim & 1 & 2 & $3(3,8 \%)$ \\
\hline Artrose & & & & Insônia & & & \\
\hline Não & 48 & 25 & $73(93,6 \%)$ & Não & 49 & 21 & $70(89,7 \%)$ \\
\hline Sim & 5 & 0 & $5(6,4 \%)$ & Sim & 4 & 4 & $8(10,3 \%)$ \\
\hline Condições pós-op & eratórias & & & Labirintite & & & \\
\hline Não & 53 & 24 & $77(98,7 \%)$ & Não & 46 & 20 & $66(84,6 \%)$ \\
\hline Sim & 0 & 1 & $1(1,3 \%)$ & Sim & 7 & 5 & $12(15,4 \%)$ \\
\hline Constipação & & & & Mobilidade físi & prejudic: & & \\
\hline Não & 50 & 21 & $71(91,0 \%)$ & Não & 46 & 22 & $68(87,2 \%)$ \\
\hline Sim & 3 & 4 & $7(9,0 \%)$ & Sim & 7 & 3 & $10(12,8 \%)$ \\
\hline Déficits proprioce & ptivos & & & Depressão & & & \\
\hline Não & 53 & 25 & $78(100 \%)$ & Não & 52 & 23 & $75(96,2 \%)$ \\
\hline Sim & 0 & 0 & 0 & Sim & 1 & 2 & $3(3,8 \%)$ \\
\hline Força diminuída $n$ & as extrem & les inferi & & Obesidade & & & \\
\hline Não & 50 & 23 & $73(93,6 \%)$ & Não & 50 & 25 & $75(96,2 \%)$ \\
\hline Sim & 3 & 2 & $5(6,4 \%)$ & Sim & 3 & 0 & $3(3,8 \%)$ \\
\hline Doença gastrointe & stinal & & & Osteoporose & & & \\
\hline Não & 53 & 25 & $78(100 \%)$ & Não & 44 & 23 & $67(85,9 \%)$ \\
\hline Sim & 0 & 0 & 0 & Sim & 9 & 2 & $11(14,1 \%)$ \\
\hline Diabetes mellitus & & & & Obstrução puln & tar crônic & & \\
\hline Não & 41 & 20 & $61(78,2 \%)$ & Não & 52 & 23 & $75(96,2 \%)$ \\
\hline Sim & 12 & 5 & $17(21,8 \%)$ & Sim & 1 & 2 & $3(3,8 \%)$ \\
\hline Dificuldade na ma & Ircha & & & Problema de co & & & \\
\hline Não & 49 & 20 & $69(85,5 \%)$ & Não & 34 & 15 & $49(62,8 \%)$ \\
\hline Sim & 4 & 5 & $9(11,5 \%)$ & Sim & 19 & 10 & $29(37,2 \%)$ \\
\hline Dificuldades audi & ivas & & & Tontura & & & \\
\hline Não & 42 & 19 & $61(78,2 \%)$ & Não & 38 & 18 & $56(71,8 \%)$ \\
\hline Sim & 11 & 6 & $17(21,8 \%)$ & Sim & 15 & 7 & $22(28,2 \%)$ \\
\hline Dificuldades visu & & & & Doença vascula & & & \\
\hline Não & 32 & 12 & $44(56,4 \%)$ & Não & 47 & 21 & $68(87,2 \%)$ \\
\hline Sim & 21 & 13 & $34(43,6 \%)$ & Sim & 6 & 4 & $10(12,8 \%)$ \\
\hline
\end{tabular}

Fonte: Dados do Autor 
Durante a investigação, foram pesquisados fatores fisiológicos que poderiam influenciar no risco de queda em idosos (Tabela 2). Os idosos foram questionados a respeito de diversos problemas relacionados à saúde. Destes, o que teve maior prevalência foi hipertensão arterial sistêmica (HAS) em
$64,1 \%$ dos idosos. Outros fatores encontrados com grande incidência foram dificuldades visuais $(43,6 \%)$, problema de coluna $(37,2 \%)$, ansiedade $(30,8 \%)$, tontura $(28,2 \%)$, falta de sono $(23,1 \%)$, diabetes mellitus e dificuldades auditivas, com o percentual de $21,8 \%$ cada.

Tabela 2- Resultados entre a associação entre fatores de risco ambientais e cognitivos e a ocorrência de quedas de idosos

\begin{tabular}{|c|c|c|c|c|c|c|c|}
\hline \multirow{2}{*}{$\begin{array}{c}\text { Fatores } \\
\text { Ambientais }\end{array}$} & \multicolumn{2}{|c|}{ Queda } & \multirow{2}{*}{ Total } & \multirow{2}{*}{$\begin{array}{c}\text { Fatores } \\
\text { Cognitivos }\end{array}$} & \multicolumn{2}{|c|}{ Queda } & \multirow{2}{*}{ Total } \\
\hline & $\begin{array}{c}\text { Não } \\
(\mathrm{n}=53)\end{array}$ & $\underset{(n=25)}{\operatorname{Sim}}$ & & & $\begin{array}{c}\text { Não } \\
(\mathrm{n}=53)\end{array}$ & $\underset{(\mathrm{n}=25)}{\operatorname{Sim}}$ & \\
\hline \multicolumn{4}{|c|}{ Móveis e objetos em excesso } & \multicolumn{3}{|c|}{ Estado mental rebaixado } & \\
\hline Não & 39 & 22 & $61(78,2 \%)$ & Não & 52 & 24 & $76(97,4 \%)$ \\
\hline Sim & 14 & 3 & $17(21,8 \%)$ & Sim & 1 & 1 & $2(2,6 \%)$ \\
\hline \multicolumn{4}{|c|}{ Ausência de material antiderrapante no banheiro } & \multicolumn{3}{|c|}{ História de quedas } & \\
\hline Não & 19 & 6 & $25(32,1 \%)$ & Não & 43 & 10 & $53(67,9 \%)$ \\
\hline Sim & 34 & 19 & $53(67,9 \%)$ & Sim & 10 & 15 & $25(32,1 \%)$ \\
\hline \multicolumn{4}{|c|}{$\begin{array}{l}\text { Ausência de material antiderrapante no box do } \\
\text { banheiro }\end{array}$} & \multicolumn{3}{|c|}{ Uso de dispositivos auxiliares } & \\
\hline Não & 13 & 10 & $23(29,5 \%)$ & Não & 53 & 24 & $77(98,7 \%)$ \\
\hline Sim & 40 & 15 & $55(70,5 \%)$ & Sim & 0 & 1 & $1(1,3 \%)$ \\
\hline \multicolumn{4}{|c|}{ Condições climáticas } & \multicolumn{3}{|c|}{ Idade igual ou acima de 65 anos } & \\
\hline Não & 48 & 21 & $69(85,5 \%)$ & Não & 25 & 7 & $32(41,0 \%)$ \\
\hline Sim & 5 & 4 & $9(11,5 \%)$ & Sim & 28 & 18 & $46(59,0 \%)$ \\
\hline \multicolumn{4}{|l|}{ Mobilização } & \multicolumn{3}{|c|}{ Morar sozinho } & \\
\hline Não & 53 & 25 & $78(100 \%)$ & Não & 50 & 25 & $75(96,2 \%)$ \\
\hline Sim & 0 & 0 & 0 & Sim & 3 & 0 & $3(3,8 \%)$ \\
\hline \multicolumn{4}{|c|}{ Pouca iluminação no quarto de dormir } & Demência & & & \\
\hline Não & 53 & 22 & $75(96,2 \%)$ & Não & 53 & 25 & $78(100 \%)$ \\
\hline Sim & 0 & 3 & $3(3,8 \%)$ & Sim & 0 & 0 & 0 \\
\hline \multicolumn{4}{|c|}{ Pouca iluminação no banheiro } & \multicolumn{3}{|c|}{ Prótese de membro inferior } & \\
\hline Não & 53 & 23 & $76(97,4 \%)$ & Não & 53 & 25 & $78(100 \%)$ \\
\hline Sim & 0 & 2 & $2(2,6 \%)$ & Sim & 0 & 0 & 0 \\
\hline \multicolumn{4}{|c|}{ Quarto não familiar } & \multicolumn{3}{|c|}{ Uso de cadeiras de roda } & \\
\hline Não & 53 & 25 & $78(100 \%)$ & Não & 52 & 25 & $77(98,7 \%)$ \\
\hline Sim & 0 & 0 & 0 & Sim & 1 & 0 & $1(1,3 \%)$ \\
\hline \multicolumn{4}{|c|}{ Tapetes espalhados pelo chão } & & & & \\
\hline Não & 47 & 16 & $63(80,8 \%)$ & & & & \\
\hline Sim & 6 & 9 & $15(19,2 \%)$ & & & & \\
\hline
\end{tabular}

Fonte: Dados do Autor

Apesar de encontrados várias doenças nos idosos entrevistados, muitas delas características da idade avançada, não houve correlação significativa entre qualquer fator fisiológico, exceto a idade (em anos), e a ocorrência de quedas (Tabela 3).

Dentre os idosos entrevistados, 32,1\% têm histórico de quedas. Destes, 15 (60,0\%) 
sofreram uma nova queda no período estudado. Dos que não têm histórico de quedas, apenas $10(18,9 \%)$ caíram nesse período (Tabela 2). Pelo ajuste do modelo de regressão Logística observou-se que há uma relação significativa entre ocorrência de queda e idosos que já sofreram quedas anteriormente $(p=0,001$; Tabela 3$)$.

Tabela 3- Resultados do modelo de regressão Logística para a associação entre fatores de risco fisiológicos, ambientais e cognitivos e a ocorrência de quedas de idosos.

\begin{tabular}{|c|c|c|c|c|c|c|}
\hline \multirow{2}{*}{ Fatores } & \multirow{2}{*}{ Estimativa } & \multirow{2}{*}{ Erro padrão } & \multirow{2}{*}{ Valor $p$} & \multirow{2}{*}{$\begin{array}{c}\text { Razão de } \\
\text { chance }\end{array}$} & \multicolumn{2}{|c|}{ IC95\% ${ }^{*}$} \\
\hline & & & & & Inferior & Superior \\
\hline \multicolumn{7}{|l|}{ FISIOLÓGICOS } \\
\hline Sexo & $-0,180$ & 0,491 & 0,714 & 0,835 & 0,319 & 2,187 \\
\hline Idade (em anos) & 0,094 & 0,037 & $0,011 * *$ & 1,099 & 1,021 & 1,182 \\
\hline so de remédios controlados & $-0,041$ & 0,511 & 0,936 & 0,96 & 0,353 & 2,610 \\
\hline Ansiedade & 0,883 & 0,514 & 0,086 & 2,418 & 0,883 & 6,621 \\
\hline Asma & 0,73 & 0,62 & 0,239 & 2,075 & 0,616 & 6,99 \\
\hline Constipação & 1,155 & 0,807 & 0,152 & 3,175 & 0,653 & 15,433 \\
\hline $\begin{array}{l}\text { Força diminuída nas } \\
\text { extremidades inferiores }\end{array}$ & 0,371 & 0,947 & 0,695 & 1,449 & 0,226 & 9,273 \\
\hline Diabetes Mellitus & $-0,158$ & 0,598 & 0,792 & 0,854 & 0,265 & 2,758 \\
\hline Dificuldades na marcha & 1,119 & 0,721 & 0,121 & 3,062 & 0,745 & 12,593 \\
\hline Dificuldades auditivas & 0,187 & 0,578 & 0,746 & 1,206 & 0,388 & 3,743 \\
\hline Dificuldades visuais & 0,501 & 0,489 & 0,305 & 1,651 & 0,633 & 4,305 \\
\hline Hipotensão ortostática & 0,400 & 0,697 & 0,566 & 1,492 & 0,381 & 5,847 \\
\hline Hipertensão arterial & $-0,365$ & 1,181 & 0,758 & 0,694 & 0,069 & 7,031 \\
\hline Incontinência urinária & $-0,025$ & 0,489 & 0,960 & 0,976 & 0,374 & 2,545 \\
\hline Insônia & 1,580 & 0,904 & 0,081 & 4,857 & 0,826 & 28,567 \\
\hline Labirintite & 0,847 & 0,754 & 0,261 & 2,333 & 0,533 & 10,221 \\
\hline Mobilidade física prejudicada & 0,496 & 0,644 & 0,441 & 1,643 & 0,465 & 5,803 \\
\hline Osteoporose & $-0,11$ & 0,737 & 0,882 & 0,896 & 0,211 & 3,800 \\
\hline Obstrução pulmonar crônica & $-0,855$ & 0,823 & 0,299 & 0,425 & 0,085 & 2,133 \\
\hline Problema de coluna & 0,176 & 0,499 & 0,723 & 1,193 & 0,449 & 3,171 \\
\hline Tontura & $-0,015$ & 0,54 & 0,978 & 0,985 & 0,342 & 2,838 \\
\hline \multicolumn{7}{|l|}{ AMBIENTAIS } \\
\hline Móveis e objetos em excesso & $-0,968$ & 0,69 & 0,161 & 0,380 & 0,098 & 1,468 \\
\hline $\begin{array}{c}\text { Material antiderrapante no } \\
\text { banheiro }\end{array}$ & 0,571 & 0,549 & 0,298 & 1,770 & 0,603 & 5,190 \\
\hline $\begin{array}{c}\text { Material antiderrapante no } \\
\text { box do banheiro }\end{array}$ & $-0,718$ & 0,518 & 0,166 & 0,488 & 0,177 & 1,346 \\
\hline Condições climáticas & 0,619 & 0,514 & 0,228 & 1,857 & 0,679 & 5,081 \\
\hline Tapetes espalhados pelo chão & 1,483 & 0,601 & $0,014 * *$ & 4,406 & 1,356 & 14,318 \\
\hline \multicolumn{7}{|l|}{ COGNITIVOS } \\
\hline Estado mental rebaixado & 0,773 & 1,436 & 0,590 & 2,167 & 0,13 & 36,121 \\
\hline Histórico de quedas & 1,864 & 0,538 & $0,001^{* *}$ & 6,45 & 2,245 & 18,53 \\
\hline
\end{tabular}


*IC95\%: intervalo de $95 \%$ de confiança da razão de chance. **Significativo a 5\%.

Individualmente, ao nível de significância de 5\%, as únicas variáveis que foram significativas foram presença de tapetes espalhados pelo chão da moradia do idoso, o histórico de quedas anteriores e a idade do idoso. A variável idade do idoso foi significativa, quando dicotomizada entre idosos com idades entre 60 e 65 anos e idosos com idades maiores e iguais a 65 anos, e também sem dicotomização.

Nenhuma das variáveis estudadas nos fatores fisiológicos foi significativa. Observando-se a razão de riscos das variáveis significativas é possível concluir que a presença de tapetes espalhados pelo chão na moradia dos idosos aumenta em 4,4 vezes o risco de queda dos idosos. Além disso, idosos com histórico de quedas anteriores possuem aproximadamente 6,5 vezes mais risco de quedas do que os idosos sem histórico de quedas. A partir da variável idade dicotomizada é possível concluir que os idosos com idade maior ou igual a 65 anos têm aproximadamente 8,2 vezes mais risco de sofrer quedas do que o idoso com idade entre 60 e 65 anos. Analisando-se a razão de risco da variável idade, sem dicotomização, é possível concluir que a cada ano, o risco de queda dos idosos aumenta aproximadamente 1,1 vezes, ou seja, a cada ano o idoso aumenta o risco de queda em 10\%. Com $95 \%$ de confiança é possível concluir que a presença de tapetes espalhados pela moradia do idoso aumenta entre 1,4 e 14,3 vezes o risco de queda do idoso. A presença de quedas anteriores aumenta entre 2,2 e 18,5 vezes o risco de queda dos idosos, ou seja, idosos que já caíram possuem entre 2,2 e 18,5 vezes mais risco de queda do que os idosos que não caíram. Os idosos com mais de 65 anos possuem entre 1,7 e 38,3 vezes mais risco de quedas do que os idosos com idade inferior a 65 anos e, o aumento de um ano na idade dos idosos causa um aumento entre 1,02 e 1,18 vezes do risco de queda dos idosos (Tabela 3).

\section{DISCUSSÃO}

Os dados obtidos no estudo demonstraram que a prevalência de idosos que sofreram quedas, está próxima da média nacional, que é de $30 \%$ (PERRACINI; RAMOS, 2002; SIQUEIRA et al.,2011). Na amostra estudada observa-se que $32,1 \%$ dos entrevistados sofreram quedas.

Percebe-se que as quedas fazem com que os idosos percam sua autonomia e a qualidade de vida, e isso repercute em seus cuidadores, principalmente em seus familiares, mudando suas rotinas para adaptar-se aos cuidados especiais que os 
idosos necessitam após a queda (COUTINHO; SILVA, 2002; FABRÍCIO; RODRIGUES; JÚNIOR, 2004). Quedas podem provocar fraturas, sendo que após a fratura o risco de óbito aumenta significativamente (COUTINHO; BLOCH; COELI, 2012; MONTEIRO; FARO, 2010).

No grupo avaliado uma maior predominância do sexo feminino. Quando se realiza pesquisas em programas de atenção à saúde, a presença de mulheres é sempre maior, pois elas procuram mais os serviços de saúde. Isso é ainda mais marcante na população idosa (MACHADO et al.,2009) Apesar da predominância do sexo feminino em nosso estudo, não houve maior prevalência desse gênero em relação ao risco de queda em idosos, embora outros estudos destaquem o risco em idosos do sexo feminino como superior. (FHON et al., 2013; GOMES et al., 2014).

Em percentual, os idosos do sexo masculino foram os que mais tiveram queda nesse último ano, o que se equipara ao estudo de Pinho et al. Entretanto, analisando-se os resultados não se nota nenhuma relação significativa em o idoso ser do sexo feminino ou masculino e a ocorrência de quedas.

Poucos trabalhos são publicados a respeito de medicamentos como fator de risco para fratura por queda em idosos, apesar da correlação significativa nos trabalhos existentes (COUTINHO; SILVA, 2002; HAMRA; RIBEIRO; MIGUEL, 2007).
Entretanto, em nosso trabalho, quanto ao uso de medicamentos e o risco de quedas, não houve relação significância $(p=0,936), \quad$ o mesmo foi encontrado por Fabrício, Rodrigues e Junior (2004).

$\mathrm{Na}$ análise dos resultados obtidos, foi verificado que o fator cognitivo histórico de quedas tem uma relação direta com o risco de queda em idosos. Tal fato pode ser evidenciado porque idosos com histórico de quedas apresentam maior comprometimento na avaliação do equilíbrio funcional em relação àqueles sem quedas (GOLÇALVES; RICCI; COIMBRA, 2009).

O medo de queda, principalmente, após experiências com consequências mais graves, pode conduzir a uma restrição de atividades levando à redução do convívio social e ao isolamento, o que afeta negativamente a qualidade de vida desses idosos (ANTES et al., 2013).

Com o envelhecimento há a modificação do perfil epidemiológico e demográfico brasileiro, com aumento significativo das doenças denominadas não transmissíveis (MONTEIRO; FARO, 2010). Porém, os resultados demonstraram que vários fatores fisiológicos não influenciaram significativamente na ocorrência de queda em idosos. O aumento idade influencia significativamente na ocorrência de queda de idosos (CARVALHO; LUCKOW; SIQUEIRA, 2011; SIQUEIRA et al., 2011), vários são as características que alteram com 
o aumento da idade do idoso, entre elas a incapacidade físico-funcional expressa em termos de comprometimento na realização de algumas atividades de vida diária (MENEZES; BACHION, 2008).

Entre os fatores ambientais, a presença de Tapetes espalhados pelo chão influencia significativamente na ocorrência de queda de idosos. Estudos mostram que o lar do idoso é o principal cenário das quedas e vários são os facilitadores das quedas encontrados no domicílio, entre eles, tapetes soltos (FERRER; PERRACINE; RAMOS, 2004; MONTEIRO; FARO, 2010).

\section{CONSIDERAÇÕES FINAIS}

Concluiu-se que o histórico de quedas anteriores, o aumento idade e a presença de tapetes espalhados pelo chão influenciam significativamente na ocorrência de queda de idosos, mostrando a necessidade de medidas de atenção básica a esses fatores por parte de programas assistenciais de saúde.

Os fatores sexo, o uso de medicamentos controlados, presença hipertensão arterial e a presença de labirintite não influenciam no risco de quedas, assim como o restante das variáveis, foram não significativas na ocorrência de queda.

Faz-se necessário mais uma vez a atenção redobrada no cotidiano destes pacientes para que se possam evitar novas quedas e fraturas, e uma vez que foram identificados os idosos que sofreram quedas, a vigilância destes torna-se fundamental para não haver novas ocorrências, e para possível continuação deste trabalho, comparando-se os resultados em dois períodos.

\section{REFERÊNCIAS}

ANTES, D. L. et al. Medo de queda recorrente e fatores associados em idosos de Florianópolis, Santa Catarina, Brasil. Cad. Saúde Pública, Rio de Janeiro, v. 29, n. 4, p.758-768, abr. 2013.

BRASIL. Ministério da Saúde. Sistemas de Informação. DATASUS. [Acessado 2012 set 12]. Disponível em:

$<$ http://tabnet.datasus.gov.br/cgi/tabcgi.exe?ib ge/cnv/popuf.def $>$.

CARVALHO, M. P.; LUCKOW, E. L. T.; SIQUEIRA, F.V. Quedas e fatores associados em idosos institucionalizados no município de Pelotas (RS, Brasil). Ciênc Saúde Coletiva. 2011; 16(6):2945-2952.

COLLET, D. Modelling binary data. London: Chapman \& Hall, 1991. 369 p.

COUTINHO, E. S. F; SILVA, S. D. Uso de medicamentos como fator de risco para fratura grave decorrente de queda em idosos. Cad. Saúde Pública [online]. 2002; 18(5):1359- 1366.

COUTINHO, E.S.F.; BLOCH, K. V.; COELI, C. M. One-year mortality among elderly people after hospitalization due to fallrelated fractures: comparison with a control group of matched elderly. Cad. Saúde

Pública, Rio de Janeiro, 2012; 28(4):801-805.

FABRÍCIO, S. C. C.; RODRIGUES, R. A. P.; JUNIOR, M. L. C. Causas e quedas de idosos 
atendidos em hospitais públicos. Rev. Saúde

Pública. 2004; 38(1):93-99

FERRER, M. L. P.; PERRACINE , M. R.; RAMOS, L. R. Prevalência de fatores ambientais associados a quedas em idosos residentes na comunidade em São Paulo, SP. Revista Brasileira de Fisioterapia. 2004; 8(2): 149-54.

FREITAS, M. A. V.; SCHEICHER, M. E. Preocupação de idosos em relação a quedas.

Rev. Bras. Geriatr. Gerontol, Rio de Janeiro, 2008; 11(1):57-64.

FREITAS, M. G. et al. Idosos atendidos em serviços de urgência no Brasil: um estudo para vítimas de quedas e de acidentes de trânsito. Ciência \& Saúde Coletiva, Rio de Janeiro, v. 20, n. 3, p.701-712, mar. 2015.

FHON, J. R. S. et al. Prevalência de quedas de idosos em situação de fragilidade. Revista de Saúde Pública, São Paulo, v. 47, n. 4, p.266-273, 2013.

GONÇALVES, D. F. F.; RICCI, N. A.; COIMBRA, A. M. V. Equilíbrio funcional de Idosos da comunidade: comparação em relação ao histórico de quedas. Rev Bras Fisioter. 2009; 13(4): 316-323.

HAMRA, A.; RIBEIRO, M. B.; MIGUEL, O. F. Correlação entre fatura por queda em idosos e uso prévio de medicamentos. Acta

Ortop Brás. 2007; 15(3): 143-145.

HORAK, F. B. Postural orientation and equilibrium: what do we need to know about neural control of balance to prevent falls? Age and Ageing. v.35-S2, p. ii7-ii11, 2006.

INSTITUTO BRASILEIRO DE GEOGRAFIA E ESTATÍSTICA. IBGE.

Pesquisa Nacional por Amostra de Domicílios. Síntese de Indicadores. Brasília: 2010. IBGE. [Acessado 2012 set 12].

Disponível em: < http://www.ibge.gov.br/ >.
MACHADO, T. R. et al. Avaliação da presença de risco para queda em idosos. Rev. Eletrônica de Enfermagem. 2009; 11(1):3238. Disponível em:

$<$ http://www.fen.ufg.br/revista/v11/n1/v11n1a 04.htm>

MENEZES, R.L.; BACHION, M. M. Estudo da presença de fatores de riscos intrínsecos para quedas, em idosos institucionalizados. Ciência \& Saúde Coletiva. 2008; 13(4):1209-1218.

MONTEIRO, C.; FARO, A. C. M. Avaliação funcional de idoso vítima de fraturas na hospitalização e no domicílio. Rev Esc Enferm USP. $2010 ; 44(3): 719-724$.

Disponível em :

$<$ http://www.scielo.br/pdf/reeusp/v44n3/24.pd f>

NICOLUSSI, A.C. et al. Qualidade de vida em idosos que sofreram quedas: revisão integrativa da literatura. Ciência \& Saúde Coletiva. 2012; 17(3):723-730.

PERRACINI, M. R.; RAMOS, L. R. Fatores associados a quedas em um corte de idosos residentes na comunidade. Rev. Saúde Pública. 2002; 36(6):709-716.

PINHO, T. A. M. et al. Avaliação do risco de quedas em idosos atendidos em Unidade Básica de Saúde. Rev Esc Enferm USP. 2012; 46(2):320-327

SIQUEIRA et al. Prevalence of falls in elderly in Brazil:a countrywide analysis. Cad. Saúde Pública. 2011; 27(9):1819-1826.

VERAS, R. Envelhecimento populacional contemporâneo: demandas, desafios e inovações. Rev Saúde Publica. 2009; 43(3):548-554. 